\title{
Clinical impact of a strategy involving endoscopic submucosal dissection for early gastric cancer: determining the optimal pathway
}

\author{
Satoru Nonaka $\cdot$ Ichiro Oda $\cdot$ Teruo Nakaya $\cdot$ Chika Kusano $\cdot$ Haruhisa Suzuki $\cdot$ \\ Shigetaka Yoshinaga - Takeo Fukagawa - Hitoshi Katai · Takuji Gotoda
}

Received: 16 June 2010/ Accepted: 30 September 2010/Published online: 17 February 2011

(c) The International Gastric Cancer Association and The Japanese Gastric Cancer Association 2011

\begin{abstract}
Background Endoscopic submucosal dissection (ESD) is a technique developed to enable the endoscopic resection (ER) of large and ulcerative neoplastic lesions that were previously unresectable using conventional endoscopic mucosal resection (EMR). We investigated the clinical outcomes of ER of early gastric cancer (EGC) before and after the introduction of ESD, with particular attention to surgery and its potential consequences.

Methods We reviewed 2,785 consecutive surgical patients with EGC and 2,469 consecutive lesions treated by ER with curative intent between 1990 and 2005. The study was divided into an EMR period (1990-1999) and an ESD period (2000-2005). We analyzed the clinical outcomes of endoscopic and surgical resections and defined 'potentially avoidable surgery' as cases of surgery performed for lesions curable by ER.

Results The rate of potentially avoidable surgery was $3.8 \%(52 / 1,369)$ in the EMR period and $0.2 \%(3 / 1,416)$ in the ESD period $(P<0.001)$. For ER patients, the rate of overall non-curative ER was $36.9 \%$ (154/417) in the EMR group and $17.0 \%(348 / 2,052)$ in the ESD group $(P<0.001)$. The rate of non-curative ER for lesions

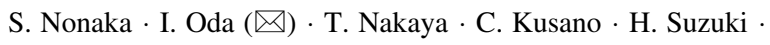

S. Yoshinaga $\cdot$ T. Gotoda

Endoscopy Division, National Cancer Center Hospital,

5-1-1 Tsukiji, Chuo-ku, Tokyo 104-0045, Japan

e-mail: ioda@ncc.go.jp

C. Kusano - T. Gotoda

Gastroenterology and Hepatology, National Center

for Global Health and Medicine, Tokyo, Japan

T. Fukagawa · H. Katai

Gastric Surgery Division, National Cancer Center Hospital,

Tokyo, Japan
\end{abstract}

defined as having 'positive or difficult to estimate horizontal margins only' decreased significantly, from $26.1 \%$ $(109 / 417)$ in the EMR group to $1.4 \%(29 / 2,052)$ in the ESD group $(P<0.001)$. Conversely, the rate of non-curative ER for lesions defined as having 'possible lymph node metastasis' significantly increased in the ESD group $(15.5 \% ; 319 / 2,052)$ compared to that in the EMR group (10.8\%; 45/417) $(P<0.01)$.

Conclusions The application of a pathway involving ESD resulted in a significant decrease in the rate of potentially avoidable surgery, highlighting the advantages associated with performing ESD.

Keywords Early gastric cancer - Lymph node metastasis · Endoscopic submucosal dissection · Potentially avoidable surgery $\cdot$ Non-curative endoscopic resection
Abbreviations
ER Endoscopic resection
EGC Early gastric cancer
EMR Endoscopic mucosal resection
ESD Endoscopic submucosal dissection
sm2 Submucosal deep invasion
sm1 Submucosal superficial invasion

\section{Introduction}

Therapeutic endoscopic resection (ER) has been performed for early gastric cancer (EGC) since the mid 1980s and is now accepted as the standard treatment for those patients with negligible risk of lymph node metastasis [1-8]. The conventional method by which EGCs were removed was by endoscopic mucosal resection (EMR). The limitations 
of applying EMR to all potentially endoscopically resectable lesions were size, location, and scarring from previous ulceration, so that only piecemeal removal was possible in such cases [9-11]. Unfortunately, piecemeal resection of EGC is associated with both difficulties in accurate histological assessment and a higher rate of local recurrence $[12,13]$. Consequently, surgery was often chosen as the initial preferred method of treatment for lesions which were difficult to resect by EMR and those associated with difficulty in estimation of tumor depth.

A major breakthrough was achieved at the turn of the twenty-first century, with the advent of endoscopic submucosal dissection (ESD) [14-20]. ESD is a technique developed to enable the resection of large and ulcerative lesions, regardless of tumor location, that are unable to be removed using the conventional EMR procedure. The other major advantage of ESD is its ability to achieve a higher rate of en-bloc resection, thus providing more accurate histological assessment as compared to EMR [12, 21]. For the aforementioned reasons, ESD has translated into lower rates of local recurrence of gastric cancer as compared with EMR [22, 23]. The gastric cancer treatment guidelines of the Japanese Gastric Cancer Association for lesions that are considered curative by EMR are shown in Table 1 [24]. Based on the risk of lymph node metastasis determined from a large cohort of surgically treated cases of EGCs, ESD is now regarded as a curative procedure for lesions selected using the National Cancer Center expanded criteria (Table 2) [25].

Table 1 JGCA guideline criteria for endoscopic resection

Differentiated adenocarcinoma

Intramucosal cancer

$\leq 20 \mathrm{~mm}$ in size without ulceration

JGCA Japanese Gastric Cancer Association

Table 2 NCC expanded histopathological criteria for curative endoscopic resection

\begin{tabular}{l}
\hline Early gastric cancer with negligible risk of lymph node metastasis \\
Differentiated adenocarcinoma \\
No lymphatic or venous invasion \\
Intramucosal cancer regardless of tumor size without ulceration \\
Or intramucosal cancer $\leq 30 \mathrm{~mm}$ in size with ulceration \\
Or submucosal superficial cancer $(\mathrm{sm} 1) \leq 30 \mathrm{~mm}$ in size \\
Resection margin \\
Tumor-free horizontal margin \\
Tumor-free vertical margin
\end{tabular}

NCC National Cancer Center
An important advantage of ESD is that it can also be considered as improving diagnostic assessment due to the suboptimal accuracy of the endoscopic staging of EGC, which is sometimes difficult because EGC shows unclear margins due to gastritis, and depth diagnosis is not always accurate [26-28]. Thus, the use of ESD has enabled us to achieve enhanced diagnosis of lesions where it may have been difficult to estimate the tumor depth or where there was a technical difficulty in resection with EMR. The treatment strategy in which additional surgery is performed after confirmation of the histological assessment of the ER specimen has already been established as one of the therapies for EGC [29-31]. We hypothesized that ESD might reduce the rate of potentially avoidable surgery by its improvement of diagnostic and therapeutic capacity compared to that of EMR. We retrospectively investigated the relationship between the surgical and endoscopic treatment of EGC before and after the introduction of ESD, with particular attention to the rate of surgical resection and its potential consequences.

\section{Patients and methods}

We retrospectively reviewed the clinical records and endoscopic and histological reports of 2,785 consecutive patients with EGC treated by surgery with curative intent and 3,102 consecutive EGC lesions treated by ER at the National Cancer Center Hospital, Tokyo, between 1990 and 2005. Informed consent was obtained from all patients in accordance with the institutional protocol. Our primary aim in this study was to retrospectively compare the rate of potentially avoidable surgery before and after the introduction of ESD and to compare the rates of non-curative ER and rates of complications between the EMR and ESD groups. All patients and lesions were discussed and the treatment strategies were determined in weekly multidisciplinary conferences involving endoscopists, surgeons, radiologists, and pathologists. The study was divided into an EMR period (1990-1999), during which the main endoscopic modality of treatment for EGC was EMR, based on the guideline criteria of the Japanese Gastric Cancer Association (Table 1) [24] and an ESD period (2000-2005), during which ESD became the predominant method by which EGCs were endoscopically resected, based on the National Cancer Center expanded criteria (Table 2) [25].

For surgical patients, we defined cases of 'potentially avoidable surgery' as those cases with surgically resected histopathological specimens within the guideline criteria of the Japanese Gastric Cancer Association [24]. In other words, the patients with potentially avoidable surgery were those who underwent surgery for lesions curable by ER. 
In the ER patients, 2,469 lesions, after exclusions, were treated by ER with curative intent; 417 lesions from the EMR group included only those lesions that were treated by EMR during the EMR period, while 2,052 lesions from the ESD group involved only those lesions that were treated by ESD during the ESD period. Another 248 lesions that were treated by ESD in the EMR period and 90 lesions that were treated by EMR in the ESD period, all with curative intent, were excluded from this study (Fig. 1). In addition, other EGCs were excluded from this study because ERs were performed for palliative purposes or because the ERs were performed for residual/recurrent lesions from previous endoscopic treatments. Palliative ERs were performed in patients who refused or were unfit for surgery because of comorbidities and for those lesions found during pre-therapeutic staging to have submucosal deep invasion (sm2) or deeper invasion, as well as those lesions with undifferentiated adenocarcinomas as revealed by biopsies. Palliative ERs included 191 lesions (150 by ESD and 41 by EMR) and residual/recurrent ERs included 104 lesions (100 by ESD and four by EMR) during each respective period (Fig. 1).

The curability of ER was divided into categories of curative and non-curative; the non-curative category included lesions that could not be precisely evaluated histologically based on the National Cancer Center expanded criteria and the tumor margins [25]. Non-curative ER was separated into two groups based on histological results: 'non-curative with positive or difficult to estimate horizontal margins only' and 'non-curative with a possible risk of lymph node metastasis irrespective of horizontal margin', based on submucosal deep invasion (sm2: $\geq 500 \mu \mathrm{m})$, positive lymphatic and/or venous invasion, intramucosal cancer more than $3 \mathrm{~cm}$ in size in the presence of ulceration, submucosal superficial invasion $(\mathrm{sm} 1:<500 \mu \mathrm{m})$ in a lesion greater than $3 \mathrm{~cm}$ in size, predominantly undifferentiated type adenocarcinoma, and positive vertical margin (Table 3). Therefore, non-curative ERs with a possible risk of lymph node metastasis were cases of ER carried out in patients who went on to require additional surgery. In other words, these patients were those who underwent ER for lesions curable by surgery. Complications including perforation and delayed bleeding that required blood transfusion were also investigated in the EMR and ESD groups.

Clinical outcomes were analyzed using the $\chi^{2}$ test and Fisher's exact test (Statview; Abacus Concepts, Berkeley, CA, USA), and $P<0.05$ was considered statistically significant.
Fig. 1 Outline of the study, including rates of potentially avoidable surgery and noncurative endoscopic resection based on the histological results. $E G C$ Early gastric cancer, $E M R$ endoscopic mucosal resection, $E S D$ endoscopic submucosal dissection, $E R$ endoscopic resection, $L N M$ lymph node metastasis, $P H M$ positive or difficult to estimate horizontal margin

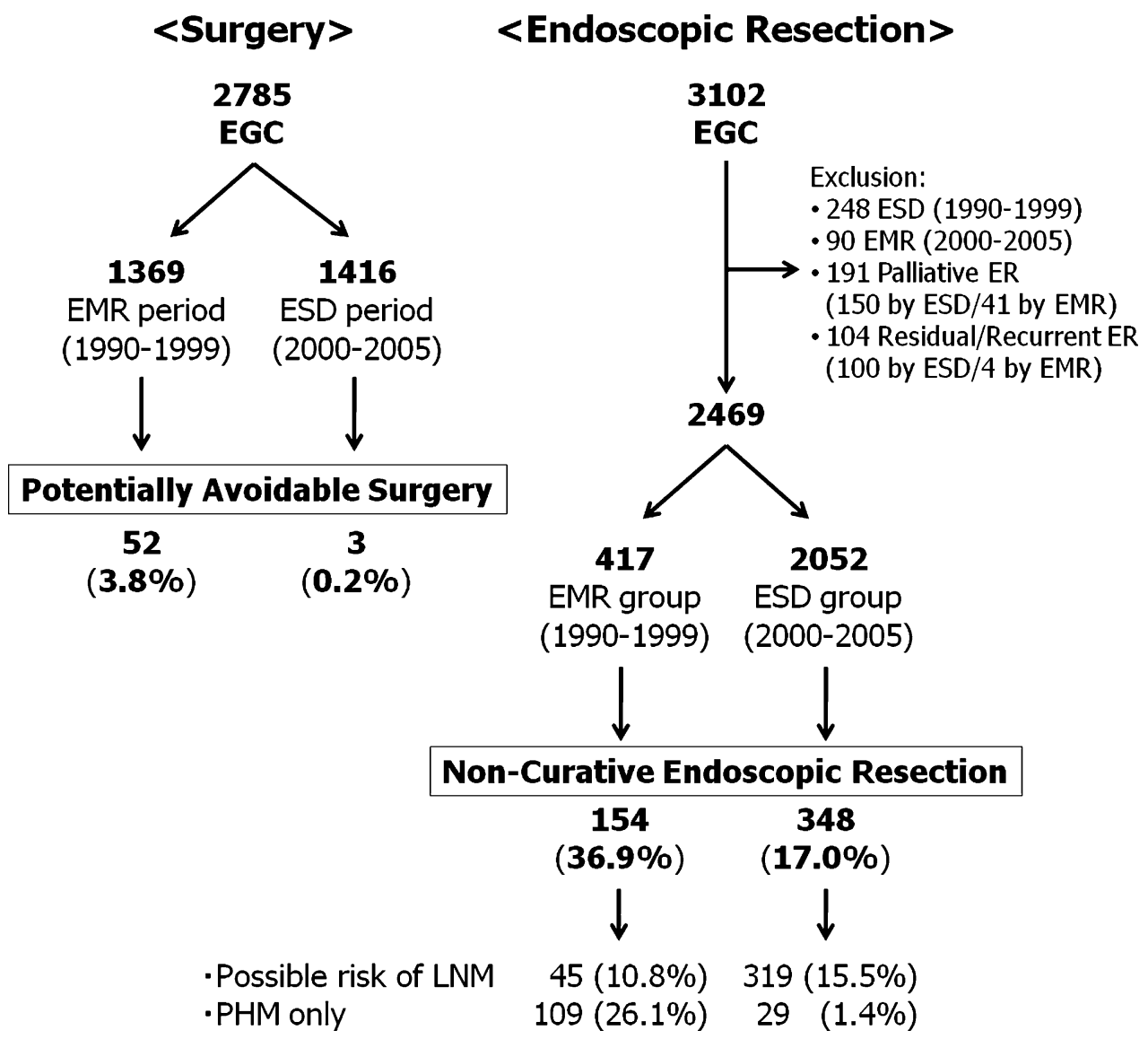

<Surgery>

2785

1369

1416

ESD period

(2000-2005)

3

$(3.8 \%) \quad(0.2 \%)$
<Endoscopic Resection>

3102

Exclusion:

- 248 ESD (1990-1999)

90 EMR (2000-2005)

(150 by ESD/41 by EMR)

- 104 Residual/Recurrent ER

( 100 by ESD/4 by EMR)

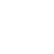


Table 3 Non-curative endoscopic resection

Non-curative with possible risk of lymph node metastasis

Submucosal deep invasion (sm2)

Positive lymphatic and/or venous invasion

Intramucosal cancer $>30 \mathrm{~mm}$ in size with ulceration

Submucosal superficial invasion $(\mathrm{sm} 1)>30 \mathrm{~mm}$ in size

Predominantly undifferentiated type adenocarcinoma

Positive vertical margin

Non-curative with positive or difficult to estimate horizontal margins only

Table 4 Rates of potentially avoidable surgery

\begin{tabular}{llll}
\hline & $\begin{array}{l}\text { EMR period } \\
(1990-1999)\end{array}$ & $\begin{array}{l}\text { ESD period } \\
(2000-2005)\end{array}$ & $P$ \\
\hline Treated surgically & 1,369 & 1,416 & \\
Guideline lesion & $52(3.8 \%)$ & $3(0.2 \%)$ & $<0.001$ \\
Technical difficulty & 21 & 0 & $<0.001$ \\
Incorrect assessment & 31 & 3 & $<0.001$ \\
\hline
\end{tabular}

$E M R$ endoscopic mucosal resection, ESD endoscopic submucosal dissection

\section{Results}

Potentially avoidable surgery

The study results are outlined in Fig. 1 . The rate of potentially avoidable surgery was $3.8 \%(52 / 1,369)$ in the EMR period and $0.2 \%(3 / 1,416)$ in the ESD period $(P<0.001)$ (Table 4$)$. There were two possible contributory factors to potentially avoidable surgery: technical difficulty with ER and incorrect pre-therapeutic assessment of EGC. EMR was not possible in 21 patients where technical difficulty arose from there being a remnant stomach due to prior surgery; scarring from previous ulceration close to the lesion; and the location of the lesion, in particular those very close to the pylorus and the gastroesophageal junction. Thirty-one patients did not undergo EMR due to incorrect pre-therapeutic endoscopic findings suggesting submucosal invasion and unclear margins. In the ESD group, all attempted lesions were treated successfully with ESD, and, in the ESD period, there were three surgical patients with incorrect preoperative assessments with lesions thought to have submucosal invasion (Table 4).

Non-curative ER with possible risk of lymph node metastasis and positive or difficult to estimate horizontal margins only

The rate of overall non-curative ER was $36.9 \%$ (154/417) in the EMR group and $17.0 \%(348 / 2,052)$ in the ESD group
Table 5 Rates of non-curative endoscopic resection

\begin{tabular}{llll}
\hline & $\begin{array}{l}\text { EMR group } \\
\%(n=417)\end{array}$ & $\begin{array}{l}\text { ESD group } \\
\%(n=2,052)\end{array}$ & $P$ \\
\hline $\begin{array}{l}\text { Non-curative with possible } \\
\text { LNM }\end{array}$ & $10.8(45)$ & $15.5(319)$ & $<0.01$ \\
Non-curative with PHM only & $26.1(109)$ & $1.4(29)$ & $<0.001$ \\
Total & $36.9(154)$ & $17.0(348)$ & $<0.001$ \\
\hline
\end{tabular}

$E M R$ endoscopic mucosal resection, ESD endoscopic submucosal dissection, $L N M$ lymph node metastasis, $P H M$ positive or difficult to estimate horizontal margin

Table 6 Causes of non-curative endoscopic resection

\begin{tabular}{|c|c|c|c|}
\hline & $\begin{array}{l}\text { EMR group } \\
\%(n=417)\end{array}$ & $\begin{array}{l}\text { ESD group } \\
\%(n=2,052)\end{array}$ & $P$ \\
\hline sm2 cancer & $8.9(37)$ & $7.5(153)$ & NS \\
\hline $\begin{array}{l}\text { Positive lymphatic and/or } \\
\text { venous invasion }\end{array}$ & $5.3(22)$ & $5.4(110)$ & NS \\
\hline $\begin{array}{l}\text { Intramucosal cancer } \\
>30 \mathrm{~mm} \text { in size with } \\
\text { ulceration }\end{array}$ & $0(0)$ & $1.7(34)$ & $<0.004$ \\
\hline sm 1 cancer $>30 \mathrm{~mm}$ in size & $0(0)$ & $2.3(48)$ & $<0.0003$ \\
\hline $\begin{array}{l}\text { Predominantly } \\
\text { undifferentiated type }\end{array}$ & $1.4(6)$ & $3.8(79)$ & $<0.01$ \\
\hline Positive vertical margin & $4.6(19)$ & $2.2(46)$ & $<0.007$ \\
\hline Positive horizontal margin & $31.4(131)$ & $3.0(62)$ & $<0.001$ \\
\hline
\end{tabular}

In some patients there was more than one cause

$E M R$ endoscopic mucosal resection, ESD endoscopic submucosal dissection, $s m 2$ submucosal deep invasion, $s m 1$ submucosal superficial invasion, NS not significant

$(P<0.001)$ (Fig. 1) (Table 5). Reasons for non-curative ER are summarized in Table 6 . The rates of sm2 invasion and positive lymphatic and/or venous involvement did not differ between the two groups. However, rates of intramucosal cancer more than $3 \mathrm{~cm}$ in size with ulceration, sm1 lesions more than $3 \mathrm{~cm}$ in size, and predominantly undifferentiated type adenocarcinoma in the ESD group significantly increased compared to those in the EMR group. The rate of positive vertical margins significantly decreased in the ESD group. In Table 6, we have listed the causes of non-curative endoscopic resection. Lesions considered non-curative with possible risk of lymph node metastasis may have been considered as such for one or a combination of overlapping criteria. To put this another way, the rate of non-curative ER with possible risk of lymph node metastasis regardless of horizontal margin increased in the ESD group $(15.5 \% ; 319 / 2,052)$ compared to that in the EMR group $(10.8 \% ; 45 / 417)(P<0.01)$ (Table 5). Conversely, the rate of non-curative ER with positive or difficult to estimate horizontal margins only dramatically decreased in the ESD group $(1.4 \% ; 29 / 2,052)$ 
compared to that in the EMR group $(26.1 \% ; 109 / 417)$ $(P<0.001)($ Table 5$)$.

\section{Complications}

The rate of perforation in the EMR group $(6.0 \% ; 25 / 417)$ was significantly higher compared to that in the ESD group $(3.0 \% ; 62 / 2,052) \quad(P<0.003)$. All perforations were detected endoscopically during the procedure, except for one patient in the ESD group with a delayed perforation who had a gastric tube after esophagectomy. Seven patients in the EMR group and one patient in the ESD group underwent emergency surgery because the perforations were difficult to manage endoscopically using endoclips. Blood transfusion was required in one patient in each group.

\section{Discussion}

This retrospective study shows that the rate of potentially avoidable surgery decreased significantly and the overall non-curative ER rate also decreased with the development of ESD. In the ESD group, the rate of non-curative endoscopically resected specimens with positive or difficult to estimate horizontal margins only significantly decreased compared with that in the EMR group, but the rate of noncurative ERs with possible risk of lymph node metastasis increased significantly.

The rate of potentially avoidable surgery was $3.8 \%$ (52/ $1,369)$ during the EMR period and $0.2 \%(3 / 1,416)$ during the ESD period $(P<0.001)$ (Table 4$)$. We believe this may be as a result of two factors, the technical progress of ER and improved diagnostic accuracy. The progress of ER with EMR, and now ESD, over the past two decades has involved major breakthroughs in endoscopy and has revolutionized the treatment of EGC. The advent of ESD has enabled us to achieve a higher rate of en-bloc resection in situations not possible before. These include remnant stomachs, scarring from previous gastric ulceration, and certain technically difficult locations. Despite the recent development of new technology such as narrow band and autofluorescence imaging [32, 33], there have been no significant changes in our ability to diagnose the depth of invasion of EGC [27, 28]. Other studies have reported that the endoscopic staging of EGC is not always accurate and is correct in only $80-90 \%$ of cases, even with endoscopic ultrasonography [26, 34-36]. In our study, we found that incorrect preoperative assessments such as endoscopic overstaging leading to potentially avoidable surgery dropped significantly with the use of ESD (Table 4), but we believe that the increased use of ESD for enhanced diagnosis, rather than improvements in other diagnostic modalities, resulted in this reduction.
For reference, the rate of surgery for lesions included within the National Cancer Center expanded criteria was $4.7 \%(67 / 1,416)$ during the ESD period (data not shown). These lesions consisted of 18 intramucosal cancers $>20$ $\mathrm{mm}$ without ulceration, 33 intramucosal cancers $\leq 30 \mathrm{~mm}$ in size with ulceration, and $16 \mathrm{sm} 1$ cancers $\leq 30 \mathrm{~mm}$ in size. It is believed that surgery on some of these lesions was potentially avoidable, but a direct comparison using the guideline criteria of the Japanese Gastric Cancer Association and the National Cancer Center expanded criteria cannot be made because of differences between the two sets of criteria.

The rate of non-curative ER, secondary to positive or difficult to estimate horizontal margins only, in the ESD group $(1.4 \% ; 29 / 2,052)$ significantly decreased compared to that in the EMR group $(26.1 \% ; 109 / 417)(P<0.001)$ (Table 5). This reflects the inability of EMR to resect large lesions en bloc, the lesion often being resected in multiple fragments, making it difficult to ensure complete resection [9-11]. The other main problem that arises with performing EMR, even for small lesions, is the uncertainty regarding inaccurate resection margins. Several previous articles have reported higher rates of local recurrence caused by piecemeal resection and positive tumor margins $[12,13$, 22, 23, 37]. The development of ESD has addressed these problems, as it enables an en-bloc resection with tumorfree margins.

On the other hand, the rate of non-curative ERs with possible risk of lymph node metastasis (which should ideally be managed by gastrectomy with lymph node dissection) increased in the ESD group $(15.5 \%: 319 / 2,052)$ compared to that in the EMR group (10.8\%:45/417) $(P<0.01)$ (Table 5). This five percent difference could have occurred due to several reasons, but the primary cause was most likely the increase in the number of patients who underwent diagnostic ESD for borderline lesions which were either difficult to resect technically by EMR or difficult to estimate tumor depth accurately. Specifically, the introduction of the National Cancer Center expanded criteria and the ability of ESD to resect larger lesions are two possible reasons for the increase in the number of intramucosal cancers more than $3 \mathrm{~cm}$ in size with ulceration and $\mathrm{sm} 1$ lesions more than $3 \mathrm{~cm}$ in size for which ER was undertaken. An increase in the number of lesions with predominantly undifferentiated adenocarcinoma also occurred, most likely because the heterogeneity of gastric carcinoma may increase in larger-size lesions. Thus, this five percent rise in the rate of non-curative ERs with possible risk of lymph node metastasis has to be weighed against the potential advantages in undertaking ESD and the significantly reduced rate of potentially avoidable surgery. Oda et al. [31] reported that the actual rate of lymph node metastases, as determined from surgically resected 
specimens, in a group of cases of 'non-curative ESD with possible risk of lymph node metastasis', was $6.3 \%$. This emphasizes the fact that this cohort of patients should receive additional surgery.

In the present study, the rate of perforation in the EMR group $(6.0 \%)$ was significantly higher compared to that in the ESD group (3.0\%) although it is widely recognized that the rate of perforation with ESD is higher than that with EMR [22]. There is no evident explanation for this result, but one possible reason may be that EMR procedures were performed more aggressively because of curative intent in the EMR group.

The surgically resected stomach never returns to its natural state. Currently, the pathway whereby we use ESD as the optimal therapeutic strategy for the treatment of EGC seems to reduce the rate of potentially avoidable surgery and allows us to more appropriately select those cases that would benefit from additional surgery, as it enables more accurate histological assessment, particularly in difficult EGC cases. As a result, this pathway has brought about major benefits for patients by reducing potentially avoidable surgery, because with this strategy the final diagnosis is obtained with higher reliability due to precise feedback from histological assessments. However, it would be prudent to advise caution in performing ESD for EGC unless the indications have been carefully reviewed in the individual to ensure that the $\mathrm{EGC}$ is within the established selection criteria. We would emphasize that recognition of resectability and curability are two very different matters. It is also important to recognize the role of ESD in providing enhanced diagnostic information, thus contributing to the optimal therapy being undertaken for the appropriate indication.

\section{Limitations}

This study was retrospective and there were differences in criteria for ER between the two groups. In addition, the transitional phase was at the turn of the twenty-first century, but it was not clearly delineated as both procedures were being used at that time. However, we believe that by analysis by procedure (EMR and ESD) we have minimized the impact of this last factor.

\section{Conclusions}

We believe that a pathway of undertaking ESD in lesions where it may be difficult to estimate the depth of invasion and in technically difficult cases results in a significant decrease in the rate of potentially avoidable surgery, this being due to the advantages associated with not only a potentially curative procedure, but also one which provides enhanced diagnostic information and consequently enables more appropriate therapy.

Acknowledgments We thank Dr. Sunil Dolwani and Dr. Marcus Chin for their writing assistance.

Conflict of interest None.

\section{References}

1. Tada M, Shimada M, Murakamai F, Mizumachi M, Arima K, Yanai H. Development of strip-off biopsy (in Japanese with English abstract). Gastroenterol Endosc. 1984;26:833-9.

2. Tada M, Murakami A, Karita M, Yanai H, Okita K. Endoscopic resection of early gastric cancer. Endoscopy. 1993;25: $445-50$.

3. Hirao M, Masuda K, Asanuma T, Naka H, Noda K, Matsuura K, et al. Endoscopic resection of early gastric cancer and other tumors with local injection of hypertonic saline-epinephrine. Gastrointest Endosc. 1988;34:264-9.

4. Inoue H, Takeshita K, Hori H, Muraoka Y, Yoneshima H, Endo M. Endoscopic mucosal resection with a cap-fitted panendoscope for esophagus, stomach, and colon mucosal lesions. Gastrointest Endosc. 1993;39:58-62.

5. Akiyama M, Ota M, Nakajima H, Yamagata K, Munakata A. Endoscopic mucosal resection of gastric neoplasms using a ligating device. Gastrointest Endosc. 1997;45:182-6.

6. Tanabe S, Koizumi W, Kokutou M, Imaizumi H, Ishii K, Kida M, et al. Usefulness of endoscopic aspiration mucosectomy as compared with strip biopsy for the treatment of gastric mucosal cancer. Gastrointest Endosc. 1999;50:819-22.

7. Rembacken BJ, Gotoda T, Fujii T, Axon AT. Endoscopic mucosal resection. Endoscopy. 2001;33:709-18.

8. Soetikno R, Kaltenbach T, Yeh R, Gotoda T. Endoscopic mucosal resection for early cancers of the upper gastrointestinal tract. J Clin Oncol. 2005;23:4490-8.

9. Korenaga D, Haraguchi M, Tsujitani S, Okamura T, Tamada R, Sugimachi K. Clinicopathological features of mucosal carcinoma of the stomach with lymph node metastasis in eleven patients. $\mathrm{Br}$ J Surg. 1986;73:431-3.

10. Ell C, May A, Gossner L, Pech O, Gunter E, Mayer G, et al. Endoscopic mucosal resection of early cancer and high-grade dysplasia in Barrett's esophagus. Gastroenterology. 2000;118: $670-7$.

11. Tanabe S, Koizumi W, Mitomi H, Nakai H, Murakami S, Nagaba $\mathrm{S}$, et al. Clinical outcome of endoscopic aspiration mucosectomy for early stage gastric cancer. Gastrointest Endosc. 2002;56: 708-13.

12. Eguchi T, Gotoda T, Oda I, Hamanaka H, Hasuike N, Saito D. Is endoscopic one-piece mucosal resection essential for early gastric cancer? Dig Endosc. 2003;15:113-6.

13. Tanabe S, Koizumi W, Higuchi K, Sasaki T, Nakatani K, Hanaoka N, et al. Clinical outcomes of endoscopic oblique aspiration mucosectomy for superficial esophageal cancer. Gastrointest Endosc. 2008;67:814-20.

14. Gotoda T, Kondo H, Ono H, Saito Y, Yamaguchi H, Saito D, et al. A new endoscopic mucosal resection procedure using an insulation-tipped electrosurgical knife for rectal flat lesions: report of two cases. Gastrointest Endosc. 1999;50:560-3.

15. Yamamoto H, Yube T, Isoda N, Sato Y, Sekine Y, Higashizawa $\mathrm{T}$, et al. A novel method of endoscopic mucosal resection using sodium hyaluronate. Gastrointest Endosc. 1999;50:251-6. 
16. Ono H, Kondo H, Gotoda T, Shirao K, Yamaguchi H, Saito D, et al. Endoscopic mucosal resection for treatment of early gastric cancer. Gut. 2001;48:225-9.

17. Oyama T, Kikuchi Y. Aggressive endoscopic mucosal resection in the upper GI tract-Hook knife EMR method. Min Invas Ther Allied Technol. 2002;11:291-5.

18. Yahagi N, Fujishiro M, Kakushima N, Kobayashi K, Hashimoto T, Oka M. Endoscopic submucosal dissection for early gastric cancer using the tip of an electrosurgical snare (thin type). Dig Endosc. 2004;16:34-8.

19. Gotoda T. A large endoscopic resection by endoscopic submucosal dissection procedure for early gastric cancer. Clin Gastroenterol Hepatol. 2005;3:S71-3.

20. Ono H, Hasuike N, Inui T, Takizawa K, Ikehara H, Yamaguchi $\mathrm{Y}$, et al. Usefulness of a novel electrosurgical knife, the insulation-tipped diathermic knife-2, for endoscopic submucosal dissection of early gastric cancer. Gastric Cancer. 2008;11:47-52.

21. Oda I, Gotoda T, Hamanaka H, Eguchi T, Saito Y, Matsuda T, et al. Endoscopic submucosal dissection for early gastric cancer: technical feasibility, operation time and complications from a large consecutive series. Dig Endosc. 2005;17:54-8.

22. Oda I, Saito D, Tada M, Iishi H, Tanabe S, Oyama T, et al. A multicenter retrospective study of endoscopic resection for early gastric cancer. Gastric Cancer. 2006;9:262-70.

23. Oka S, Tanaka S, Kaneko I, Mouri R, Hirata M, Kawamura T, et al. Advantage of endoscopic submucosal dissection compared with EMR for early gastric cancer. Gastrointest Endosc. 2006;64:877-83.

24. Japanese Gastric Cancer Association. Gastric cancer treatment guideline (in Japanese). 2nd ed. Tokyo: Kanehara-Shuppan; 2004.

25. Gotoda T, Yanagisawa A, Sasako M, Ono H, Nakanishi Y, Shimoda $\mathrm{T}$, et al. Incidence of lymph node metastasis from early gastric cancer: estimation with a large number of cases at two large centers. Gastric Cancer. 2000;3:219-25.

26. Sano T, Okuyama Y, Kobori O, Shimizu T, Morioka Y. Early gastric cancer. Endoscopic diagnosis of depth of invasion. Dig Dis Sci. 1990;35:1340-4.

27. Ono H, Yoshida S. Endoscopic diagnosis of the depth of cancer invasion for gastric cancer. Stomach Intest (in Japanese with English abstract). 2001;36:334-40.
28. Yin JX, Oda I, Suzuki H, Gotoda T, Shimoda T, Saito D. Endoscopic diagnosis of gastric cancer invasion depth. Nippon Shokakibyo Gakkai Zasshi (in Japanese with English abstract). 2009;106:1603-9.

29. Korenaga D, Orita H, Maekawa S, Maruoka A, Sakai K, Ikeda T, et al. Pathological appearance of the stomach after endoscopic mucosal resection for early gastric cancer. Br J Surg. 1997;84: 1563-6.

30. Nagano H, Ohyama S, Fukunaga T, Seto Y, Fujisaki J, Yamaguchi $\mathrm{T}$, et al. Indications for gastrectomy after incomplete EMR for early gastric cancer. Gastric Cancer. 2005;8:149-54.

31. Oda I, Gotoda T, Sasako M, Sano T, Katai H, Fukagawa T, et al. Treatment strategy after non-curative endoscopic resection of early gastric cancer. Br J Surg. 2008;95:1495-500.

32. Nakayoshi T, Tajiri H, Matsuda K, Kaise M, Ikegami M, Sasaki H. Magnifying endoscopy combined with narrow band imaging system for early gastric cancer: correlation of vascular pattern with histopathology (including video). Endoscopy. 2004;36: 1080-4.

33. Uedo N, Iishi H, Tatsuta M, Yamada T, Ogiyama H, Imanaka K, et al. A novel videoendoscopy system by using autofluorescence and reflectance imaging for diagnosis of esophagogastric cancers. Gastrointest Endosc. 2005;62:521-8.

34. Yanai H, Matsumoto Y, Harada T, Nishiaki M, Tokiyama H, Shigemitsu T, et al. Endoscopic ultrasonography and endoscopy for staging depth of invasion in early gastric cancer: a pilot study. Gastrointest Endosc. 1997;46:212-6.

35. Yanai H, Noguchi T, Mizumachi S, Tokiyama H, Nakamura H, Tada M, et al. A blind comparison of the effectiveness of endoscopic ultrasonography and endoscopy in staging early gastric cancer. Gut. 1999;44:361-5.

36. Seto Y, Shimoyama S, Kitayama J, Mafune K, Kaminishi M, Aikou T, et al. Lymph node metastasis and preoperative diagnosis of depth of invasion in early gastric cancer. Gastric Cancer. 2001;4:34-8.

37. Watanabe K, Ogata S, Kawazoe S, Koyama T, Kajiwara T, Shimoda Y, et al. Clinical outcomes of EMR for gastric tumors: historical pilot evaluation between endoscopic submucosal dissection and conventional mucosal resection. Gastrointest Endosc. 2006;63:776-82. 